\title{
On the Odd Gracefulness of Cyclic Snakes With Pendant Edges
}

\author{
E. M. Badr \\ Department of Scientific Computing, Faculty of Computer Science and Informatics, \\ Benha University, Benha, Egypt \\ badrgraph@gmail.com
}

\begin{abstract}
Graceful and odd gracefulness of a graph are two entirely different concepts. A graph may posses one or both of these or neither. We present four new families of odd graceful graphs. In particular we show an odd graceful labeling of the linear $k C_{4}$-snake e $m K_{1}$ and therefore we introduce the odd graceful labeling of $k C_{4}$-snake e $m K_{1}$ ( for the general case). We prove that the subdivision of linear $k C_{3}-$ snake is odd graceful. We also prove that the subdivision of linear $k C_{3}$-snake with m-pendant edges is odd graceful. Finally, we present an odd graceful labeling of the crown graph $P_{n}$ e $m K_{1}$.
\end{abstract}

\section{KEYWORDS}

Graph theory, odd graceful labeling, path, cyclic snakes, pendant edges.

\section{INTRODUCTION}

The graphs considered here will be finite, undirected and simple. The symbols $V(G)$ and $E(G)$ will denote the vertex set and edge set of a graph $G$ respectively. $p$ and $q$ denote the number of vertices and edges of $G$ respectively.

A graph $G$ of size $q$ is odd-graceful, if there is an injection $\phi$ from $V(G)$ to $\{0,1,2, \ldots, 2 q-1\}$ such that, when each edge $x y$ is assigned the label or weight $|\phi(x)-\phi(y)|$, the resulting edge labels are $\{1,3,5, \ldots, 2 q-1\}$. This definition was introduced in 1991 by Gnanajothi [1] who proved that the class of odd graceful graphs lies between the class of graphs with $\alpha$-labelings and the class of bipartite graphs. We denote the crown graphs ( the graphs obtained by joining a single pendant edge to each vertex of $C_{n}$ ) by $C_{n} \square K_{1}$, therefore the crown graphs (the graphs obtained by joining $m$-pendant edges to each vertex of $C_{n}$ ) by $C_{n} \square m K_{1}$. Gnanajothi [1] proved that $C_{n} \square K_{1}$ is odd graceful if $n$ is even. Badr et al [2] proved that $C_{n} \square m K_{1}$ is odd graceful if $n$ is even.

A $k C_{n}-$ snake is a connected graph with $k$ blocks, each of the blocks is isomorphic to the cycle $C_{n}$, such that the block-cut-vertex graph is a path. Following [3], by a block-cut-vertex graph of a graph $G$ we mean the graph whose vertices are the blocks and cut-vertices of $G$ where two vertices are adjacent if and only if one vertex is a block and the other is a cut-vertex belonging to The block. We also call a $k C_{n}-$ snake as a cyclic snake. This graph was first introduced by

DOI : 10.5121/jgraphoc.2012.4401 
International journal on applications of graph theory in wireless ad hoc networks and sensor networks

(GRAPH-HOC) Vol.4, No.4, December 2012

Barrientos [4], as generalization of the concept of triangular snake introduced by Rosa [5].

Let $G$ be a $k C_{n}-$ snake with $k \geq 2$. Let $u_{1}, u_{2}, \ldots, u_{k-1}$ be the consecutive cut-vertices of $G$. Let $d_{i}$ be the distance between $v_{i}$ and $v_{i+1}$ in $G, 1 \leq i \leq k-2$. The string $\left(d_{1}, d_{2}, \ldots, d_{k-2}\right)$ of integers, characterizes the graph $G$ in the class of $n$-cyclic snakes. The $k C_{n}$-snake contains $q=n k$ edges and $p=(n-1) k+1$ vertices. Among these vertices, $k-1$ vertices have degree 4 and the other vertices of degree 2 .

Now, let us consider a $k C_{4}$-snake. The first non trivial quadrilateral snake is $2 C_{4}$-snake as shown in Figure 1. Two different $k C_{4}-$ snake can be constructed from a $(k-1) C_{4}-$ snake .

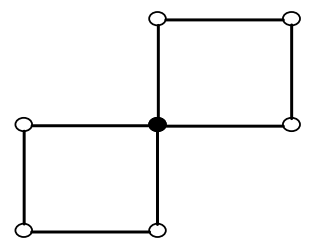

Figure 1. $2 C_{4}$-snake with a cut-vertex

Example 1.1 We can construct two different $3 C_{4}$-snake from a $2 C_{4}-$ snake, the first is with string ( 1 ) ( Figure 2a ) and the second is with string ( 2 ) ( Figure 2b ).

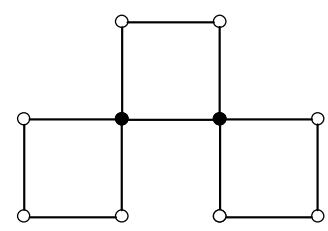

a)

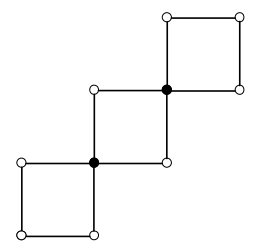

b)

Figure 2. $3 C_{4}$-snakes with string 1 and string 2 respectively.

Badr and Mousa [6] proved that $k C_{4^{-}}$snake, linear $k C_{n^{-}}$snake, even $k C_{8}-$ snake and even $k C_{12^{-}}$ snakes are odd graceful.

We denote the crown snakes ( the graphs obtained by joining $m$-pendant edges to each vertex of $k C_{n}$-snake) by $k C_{n}-$ snake e $m K_{1}$.

Definition 1.2 The $k C_{n}$-snake is called linear, if the block-cut-vertex graph of $k C_{n}$-snake has the property that the distance between any two consecutive cut-vertices is $\left\lfloor\frac{n}{2}\right\rfloor$.

In this work we present four new families of odd graceful graphs. In particular we show that an odd graceful labeling of the linear $k C_{4}$-snake e $m K_{1}$ and therefore we introduce the odd graceful labeling of $k C_{4}$-snake e $m K_{1}$ (for the general case ). We prove that the subdivision of linear $k C_{3}$-snake is odd graceful. We also prove that the subdivision of linear $k C_{3}-$ snake with 
International journal on applications of graph theory in wireless ad hoc networks and sensor networks (GRAPH-HOC) Vol.4, No.4, December 2012

$m$-pendant edges is odd graceful. Finally, we present an odd graceful labeling of the crown graph $P_{n}$ e $m K_{1}$.

\section{The Results}

Theorem 2.1 The linear $k C_{4}$-snake e $m K_{1}$ is odd graceful.

Proof. Let $G=$ linear $k C_{4}$-snake e $m K_{1}$ with the vertices $w_{i}, u_{j}$ and $v_{j}$ where $i=0,1,2$, $\ldots, k, j=0,1,2, \ldots, k$-1. In order to get the linear $k C_{4}$-snake e $m K_{1}$, we add $m$-pendant edges $w_{i}^{l}, u_{j}^{1}$ and $v_{j}^{1}$ to each vertex of $w_{i}, u_{j}$ and $v_{j}$ respectively such that $l=1,2, \ldots, m$. Now, the graph $k C_{4}-$ snake e $m K_{1}$ has the vertices $w_{i}, u_{j}, v_{j}, w_{i}{ }^{l}, u_{j}^{1}$ and $v_{j}^{1}$ such that $i=0,1,2, \ldots, k, j=0,1$, $2, \ldots, k-1$ and $l=1,2, \ldots, m$.

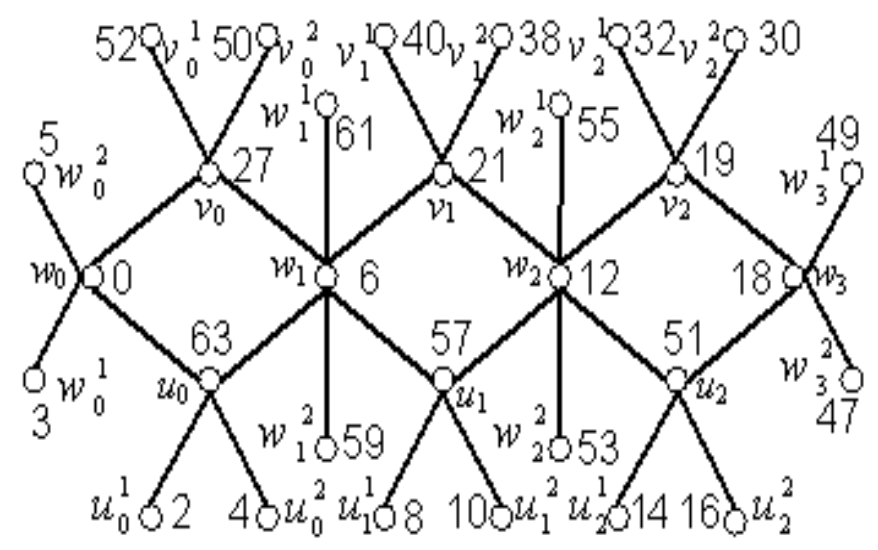

Figure $3.3 C_{4}-$ snake e $2 K_{1}$ is odd graceful

Clearly the linear $k C_{4}$-snake e $m K_{1}$ has $p=(3 k+1)(m+1)$ vertices and $q=4 k+m(3 k+1)$ edges.

Let us consider the following numbering $\phi$ of the vertices of the graph $G$ :

$$
\begin{array}{lc}
\phi\left(w_{i}\right)=2 i(m+1) & i=0,1,2, \ldots, k . \\
\phi\left(u_{i}\right)=2 q-2 i(m+1)-1 & i=0,1,2, \ldots, k . \\
\phi\left(v_{i}\right)=2 q-4 k(m+1)-2 \mathrm{i}-1 & i=0,1,2, \ldots, k . \\
\phi\left(w_{0}^{1}\right)=2 l-1, & l=1,2, \ldots, m . \\
\phi\left(w_{i}^{l}\right)=2(q-l+m-i(m+1)+1 & i=1,2, \ldots, k . \\
\phi\left(u_{i}^{1}\right)=2(l+\mathrm{i}(\mathrm{m}+1)) & i=0,1,2, \ldots, k-1,1=1,2, \ldots, m . \\
\phi\left(v_{i}^{1}\right)=4(q-2 k(m+1))-2(l+1+i(m+3)) \quad i=0,1,2, \ldots, k-1,1=1,2, \ldots, m .
\end{array}
$$


International journal on applications of graph theory in wireless ad hoc networks and sensor networks (GRAPH-HOC) Vol.4, No.4, December 2012

(a)

$$
\begin{gathered}
\operatorname{Max}_{v \in V} \phi(v)=\max \left\{\max _{0 \leq i \leq k} 2 i(m+1), \max _{0 \leq i \leq k-1} 2 q-2 i(m+1)-1, \max _{1 \leq l \leq m} 2 l-1,\right. \\
\max _{0 \leq i \leq k-1} 2 q-4 k(m+1)-2 i-1, \max _{1 \leq l \leq m} 2\left(q-l+m-i(m+1)+1, \max _{1 \leq l \leq m} 2(l+i(m+1)),\right. \\
\left.\max _{1 \leq l \leq m}^{0 \leq 1 \leq k-1} \max (q-2 k(m+1))-2(l+1+i(m+3))\right\} \\
=2 q-1, \text { the maximum value of all odds. }
\end{gathered}
$$

Thus $\phi(v) \in\{0,1,2, \ldots, 2 q-1\}$.

(b) Clearly $\phi$ is a one - to - one mapping from the vertex set of $G$ to $\{0,1,2, \ldots, 2 q-1\}$.

(c) It remains to show that the labels of the edges of $G$ are all the odd integers of the interval [1, $2 q-1]$.

The range of $\left|\phi\left(v_{i}\right)-\phi\left(w_{i}\right)\right|=\{2 q-4 k(m+1)-2 i(m+2)-1: \quad i=0,1,2, \ldots, k-1\}$

$=\{2 q-4 k(m+1)-1, \ldots, 2 q-4 k(m+1)-2 m-5, \ldots, 2 q-6 k m-8 k+2(m+2)-1\}$

The range of $\left|\phi\left(v_{i}\right)-\phi\left(w_{\mathrm{i}+1}\right)\right|=\{2 q-4 k(m+1)-2 i(m+2)-2 m-3: i=0,1,2,3, \ldots, k-1\}$

$=\{2 q-4 k(m+1)-8 k+1,2 q-4 k(m+1)-4 m-7, \ldots, 2 q-6 k m-8 k+1\}$

The range of $\left|\phi\left(u_{i}\right)-\phi\left(w_{i}\right)\right|=\{2 q-4 i(m+1)-1: i=0,1,2,3, \ldots, k-1\}$

$$
=\{2 q-1,2 q-4(m+1)-1, \ldots, 2 q-4(k-1)(m+1)-1\}
$$

The range of $\left|\phi\left(u_{i}\right)-\phi\left(w_{\mathrm{i}}\right)\right|=\{2 q-4 i(m+1)-2 m-3: i=0,1,2,3, \ldots, k-1\}$

$=\{2 q-2 m-3,2 q-6 m-7 \ldots, 2 q-4(k-1)(m+1)-2 m-3\}$

The range of $\left|\phi\left(w_{0}\right)-\phi\left(w_{0}^{1}\right)\right|=\{2 l-1: l=1,2,3, \ldots, m\}=\{1,3, \ldots, 2 m-1\}$

The range of $\left|\phi\left(w_{i}\right)-\phi\left(w_{\mathrm{i}}^{1}\right)\right|=\{2(q-l+m-2 i(m+1))+1: i=1,2,3, \ldots, k, l=1,2,3, \ldots, m\}$

$=\{2(q-1-m)-3,2(q-1-3 m)-7, \ldots, 2(q-l+m-2 k(m+1))+1\}$

The range of $\left|\phi\left(u_{i}\right)-\phi\left(u_{\mathrm{i}}^{1}\right)\right|=\{2(q-l-2 i(m+1)-1: i=0,1,2,3, \ldots, k-1 ; l=1,2,3, \ldots, m\}$

$$
=\{2(q-1)-1,2(q-1-2(m+1)-1, \ldots, 2(q-l-2(k-1)(m+1)-1\}
$$

The range of $\left|\phi\left(v_{i}\right)-\phi\left(v_{\mathrm{i}}^{1}\right)\right|=\{2(q-2 k(m+1)-l-i(m+2))-1: 0 \leq i \leq k-1 ; 1 \leq l \leq m\}=\{2(q-2 k(m+1)-1)-1$, $2(q-2 k(m+1)-1-(m+2))-1, \ldots, 2[q-2 k(m+1)-l-(k-1)(m+2)]-1\}$

Hence $\{|\phi(u)-\phi(v)|: u v \in E\}=\{1,3,5, \ldots, 2 q-1\}$ so that the graph $G$ is odd graceful.

Theorem 2.2 The $k C_{4}$-snake e $m K_{1}$ is odd graceful.

Proof. Let $\mathrm{G}=k C_{4}$-snake e $m K_{1}$. The graph $\mathrm{G}$ obtained by joining $m$-pendant edges to each vertex of $k_{4}-$ snake. Now, we are running the following steps sequentially in order to prove the Theorem. 


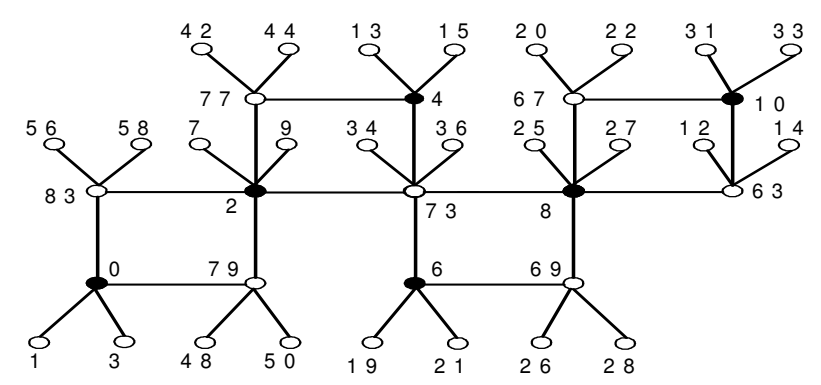

Figure $4.4 C_{4}$ e $2 K_{1}$ - snake is odd graceful

Step 1 .

Since $k C_{4}-$ snake is a bipartite graph so it has one partite set has black vertices and the other has white vertices as shown in Figure 4.

Put black vertices in a string, ordered by diagonals from left to right and inside each diagonal from bottom to top, assign to them from an arithmetic progression of difference 2, which first term is zero, counting until the last black vertex has been numbered. Similarly, put the white vertices on a string, ordered for diagonals from left to right and inside each diagonal from top to bottom. Starting with the first diagonal assign numbers from an arithmetic progression of difference 4 , which first term is $2 q$-1 where $q$ is the size of $G$, while to move to another diagonal we use an arithmetic progression of difference 2 but the arithmetic progression of difference 4 is used inside each diagonal, continuing until the last white vertex has been numbered.

Step 2 .

In this step, we are labeling the vertices of m-pendant edges which contact with black diagonals, from left to right and inside each black diagonal from bottom to top, assign to them from an arithmetic progression of difference 2, which first term is one, when to move to a new vertex of the block diagonal, the first vertex of m-pendant edges is labeled by an arithmetic progression of difference 4 , but the arithmetic progression of difference 2 has been used with the remain $(m-1)$ vertices of m-pendant edges. We move from a vertex to another of the black diagonals until the last black vertex.

Step 3 .

Finally, we are labeling the vertices of $m$-pendant edges which contact with the white diagonals, from right to left an inside each white diagonal from bottom to top, assign to them from an arithmetic progression of difference 2, which first term is z such that $z=y+2$ where $y$ is the last vertex labeling of the black diagonals, when to move to a new vertex of the white diagonal, the first vertex of $m$-pendant edges is labeled by an arithmetic progression of difference 6 , but the arithmetic progression of difference 2 has been used with the remain $(m-1)$ vertices of $m$ pendant edges. When to move to another diagonal we use an arithmetic progression of difference 4 but the arithmetic progression of difference 2 has been used with the remain $(m-1)$ vertices of $m$-pendant edges. We move from a vertex to another of the white diagonals until the last white vertex. We have the complete proof of the Theorem by running the above steps.

Theorem 2.3 The subdivision of linear $k C_{3}-$ snake is odd graceful.

Proof: We prove that the subdivision of graph $S$ ( $k C_{3}$-snake ) ( obtained by subdividing every edge of $k C_{3}$-snake exactly once ) is odd graceful. 
International journal on applications of graph theory in wireless ad hoc networks and sensor networks

(GRAPH-HOC) Vol.4, No.4, December 2012

Let $u_{0}, u_{1}, \ldots, u_{k}, v_{0}, v_{1}, \ldots, v_{k-1}$ be the vertices of the $k C_{3}$-snake. Let $y_{i}$ be the newly added vertex between $u_{i}$ and $u_{i+1}$ where $i=0,1, \ldots, k-1$ but $w_{i}$ be the newly added vertex between $u_{j}, v_{j}$ and $v_{j}, u_{j+1}$ ( from left to right) such that $i=0,1, \ldots, 2 k-1$ and $j=0,1, \ldots, k-1$ (Figure 5).

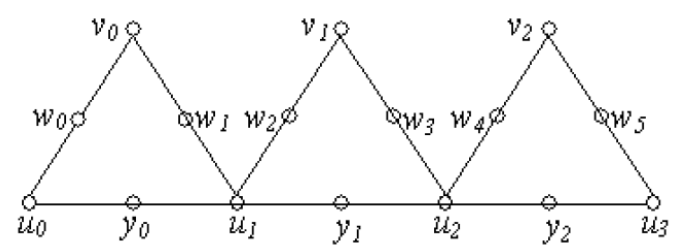

Figure 5. $S$ ( $3 C_{3}$-snake $)$ is odd graceful

Clearly $S$ ( $k C_{3}$-snake $)$ has $p=(n+2) k+1$ vertices and $q=2 n k$ edges. Let us consider the following numbering $\phi$ of the vertices of $S$ ( $k C_{3}$-snake ):

$$
\begin{array}{lrl}
\phi\left(u_{i}\right) & =4 i, & \text { where } i=0,1,2, \ldots, k . \\
\phi\left(v_{i}\right) & =4 i+2, & \text { where } i=0,1,2, \ldots, k-1 . \\
\phi\left(w_{i}\right)=2 q-2 i-1, & \text { where } i=0,1,2, \ldots, 2 k-1 . \\
\phi\left(y_{i}\right)=2 q-8 k-4 i-1, & \text { where } i=0,1,2, \ldots, k-1 .
\end{array}
$$

(a)

$$
\begin{aligned}
\operatorname{Max}_{v \in V} \phi(v)= & \max \left\{\max _{0 \leq l \leq \kappa} 4 i, \max _{0 \leq l \leq \kappa-1} 4 i+2, \max _{0 \leq l \leq 2 \kappa-1} 2 q-2 i-1, \max _{0 \leq l \leq \kappa-1} 2 q-8 k-4 i-1\right\} \\
& =2 q-1, \text { the maximum value of all odds. }
\end{aligned}
$$

Thus $\phi(v) \in\{0,1,2, \ldots, 2 q-1\}$.

(b) Clearly $\phi$ is a one - to - one mapping from the vertex set of $G$ to $\{0,1,2, \ldots, 2 q-1\}$.

(c) It remains to show that the labels of the edges of $G$ are all the odd integers of the interval [1, $2 q-1]$. Let $r$ be the number of block which has the vertex $v_{i}$ or $u_{i}$ where $i=1,2, \ldots, k$.

The range of $\left|\phi\left(u_{i}\right)-\phi\left(y_{i}\right)\right|=\{2 q-8 k-8 i-1: \quad i=0,1,2, \ldots, k-1\}$

$$
=\{2 q-8 k-1, \ldots, 2 q-8 k-9, \ldots, 2 q-16 k+7\}
$$

The range of $\left|\phi\left(y_{i}\right)-\phi\left(u_{i+1}\right)\right|=\{2 q-8 k-8 i-5: \quad i=0,1,2, \ldots, k-1\}$

$$
=\{2 q-8 k-5, \ldots, 2 q-8 k-13, \ldots, 2 q-16 k+3\}
$$

The range of $\left|\phi\left(u_{i}\right)-\phi\left(w_{i+r-1}\right)\right|=\{2 q-6 i-2 r+1: \quad i=0,1,2, \ldots, k\}$

$$
=\{2 q-1, \ldots, 2 q-7, \ldots, 2 q-8 k+1\}
$$

The range of $\left|\phi\left(v_{i}\right)-\phi\left(w_{i+r}\right)\right|=\{2 q-6 i-2 r-3: \quad i=0,1,2, \ldots, k-1\}$

$$
=\{2 q-5, \ldots, 2 q-13, \ldots, 2 q-8 k+3\}
$$

The range of $\left|\phi\left(v_{i}\right)-\phi\left(w_{i+r-1}\right)\right|=\{2 q-6 i-2 r-1: \quad i=0,1,2, \ldots, k-1\}$

$$
=\{2 q-3, \ldots, 2 q-11, \ldots, 2 q-8 k+5\}
$$


International journal on applications of graph theory in wireless ad hoc networks and sensor networks (GRAPH-HOC) Vol.4, No.4, December 2012

Hence $\{|\phi(u)-\phi(v)|: u v \in E\}=\{1,3,5, \ldots, 2 q-1\}$ so that the graph $S$ ( $k C_{3}$-snake ) is odd graceful.

Theorem 2.4 The subdivision of linear $k C_{3}$-snake with $m$-pendant edges is odd graceful.

Proof. We prove that the linear $S\left(k C_{3}-\right.$ snake) e $m K_{1}$ is odd graceful, where $S\left(k C_{3}-\right.$ snake) is obtained by subdividing every edge of $k C_{3}$-snake exactly once and $m K_{1}$ are $m$-pendant edges which join to every vertex of the graph $S\left(k C_{3}-\right.$ snake $)$.

Let $u_{1}, u_{2}, \ldots, u_{k+1}, w_{1}, w_{2}, \ldots, v_{k}$ be the vertices of the $k C_{3}$-snake . Let $y_{i}$ be the newly added vertex between $u_{i}$ and $u_{i+1}$ where $i=1, \ldots, k$ while $v_{i}$ be the newly added vertex between $u_{i}, w_{i}$ where $i=1, \ldots, k$. Finally $z_{i}$ be the newly added vertex between $w_{i}, u_{i+1}$ wher $i=1,2, \ldots, k$. In order to get the graph $G=$ linear $S\left(k C_{3}\right.$-snake $)$ e $m K_{1}$, we add $m$-pendant edges $u_{i}^{l}, y_{i}^{l}, v_{i}^{l}$, $w_{i}^{l}$ and $z_{i}^{l}$ to each vertex of $u_{i}, y_{i}, v_{i}, w_{i}$ and $z_{i}$ respectively.

Clearly the graph $G$ has $p=(5 k+1) m$ vertices and $q=(5 m+6)(k-1)+m$ edges.

Define $\varphi: V(G) \rightarrow\{0,1,2, \ldots, 2 q-1\}$ as follows:

$$
\begin{aligned}
& \phi\left(u_{i}\right)=4(m+1)(i-1) \quad 1 \leq i \leq k+1 \\
& \phi\left(v_{i}\right)=2 q-(2 m+4) i+2 m+3 \quad 1 \leq i \leq k \\
& \phi\left(w_{i}\right)=2(m+1)(2 i-1) \quad 1 \leq i \leq k \\
& \phi\left(y_{i}\right)=4(m+1)(k-i)+4 m+3 \quad 1 \leq i \leq k \\
& \phi\left(z_{i}\right)=2 q-2 i(m+2)+1 \quad 1 \leq i \leq k \\
& \phi\left(v_{i}^{l}\right)=4 i(m+1)+2(l-m-1) \quad 1 \leq i \leq k ; 1 \leq l \leq m \\
& \phi\left(w_{i}^{l}\right)=2 q-2 i(m+2)-2(l-m)+3 \quad 1 \leq i \leq k ; 1 \leq l \leq m \\
& \phi\left(z_{i}^{l}\right)=4 i(m+1)+2(k-m-1) \quad 1 \leq i \leq k ; 1 \leq l \leq m \\
& \phi\left(y_{1}^{l}\right)=2 q-2 l-(2 m+4) k-2 m-2 \quad 1 \leq l \leq m \\
& \left.\phi\left(y_{i}^{l}\right)=q-2 i(m+1)-2 l+m(k+1) \quad(k \text { odd }) ; i=2,3,4, \ldots, n \text { (i even }\right), 1 \leq l \leq m \\
& \phi\left(y_{i}^{l}\right)=q-2 i(m+1)-2 l+m(k+1)+2 \quad(k \text { odd }) ; i=2,3,4, \ldots, n(i \text { odd }), 1 \leq l \leq m \\
& \phi\left(y_{i}^{l}\right)=q-2 i(m+1)-2 l+m(k+1)+2 \quad(k \text { even }) ; i=2,3,4, \ldots, n(i \text { even }), 1 \leq l \leq m \\
& \phi\left(y_{i}^{l}\right)=q-2 i(m+1)-2 l+m(k+1) \quad(k \text { even }) ; i=2,3,4, \ldots, n(i \text { odd }), 1 \leq l \leq m \\
& \phi\left(u_{1}^{l}\right)=2 l+1 \quad 1 \leq l \leq m \\
& \phi\left(u_{i}^{l}\right)=q+2 i(m+1)-2 l-(3 m+4) k-m-1 \quad(k \text { even }) ; i=2,3,4, \ldots, n-1(n \text { even }) ; 1 \leq l \leq m \\
& \phi\left(u_{i}^{l}\right)=q+2 i(m+1)-2 l-(3 m+4) k-m+1 \quad(k \text { even }) ; i=2,3,4, \ldots, n-1(n \text { odd }) ; 1 \leq l \leq m \\
& \left.\phi\left(u_{i}^{l}\right)=q+2 i(m+1)-2 l-(3 m+4) k-m+1 \quad(k \text { odd }) ; i=2,3,4, \ldots, n-1 \text { ( } \text { even }\right) ; 1 \leq l \leq m \\
& \phi\left(u_{i}^{l}\right)=q+2 i(m+1)-2 l-(3 m+4) k-m-1 \quad(k \text { odd }) ; i=2,3,4, \ldots, n-1(\text { o odd }) ; 1 \leq l \leq m \\
& \phi\left(u_{n}^{l}\right)=2 q-2 l-(2 m+4) n+2 m+5 \quad 1 \leq l \leq m
\end{aligned}
$$

Then clearly the mapping $\phi$ is an odd graceful labeling. 
International journal on applications of graph theory in wireless ad hoc networks and sensor networks (GRAPH-HOC) Vol.4, No.4, December 2012

Theorem 2.5 The crown graph $P_{n}$ e $m K_{1}$ is odd graceful.

Proof. We can see the crown graph $G=P_{n} \square m K_{1}$ as the path $P_{n}$ which has the vertices $\quad\left(v_{1}, v_{2}\right.$, $v_{3} \ldots, v_{n}$ ) such that each vertex $v_{i}$ adjacent to $m$ pendent edges $v_{i}^{l}$ where $i=1,2,3, \ldots, n$ and $l=1$, $2,3, \ldots, m$.

Let us consider the following numbering $\phi$ of the vertices of $G$.

$$
\begin{aligned}
& \phi\left(v_{i}\right)=(m+1)(i-1), \quad(i \text { odd }), \quad i=1,2, \ldots, n . \\
& \phi\left(v_{i}\right)=2 q-(m+1)(i-1)-m, \quad(i \text { even }), \quad i=1,2, \ldots, n . \\
& \phi\left(v_{i}^{k}\right)=2 q-(m+1)(i-1)-2 l+1,(i \text { odd }), \quad i=1,2, \ldots, n, l=1,2, \ldots, m . \\
& \phi\left(v_{i}^{k}\right)=(m+1)(i+1)+2 k-(m+1), \quad(i \text { even }), \quad i=1,2, \ldots, n, \quad l=1,2, \ldots, m .
\end{aligned}
$$

(a)

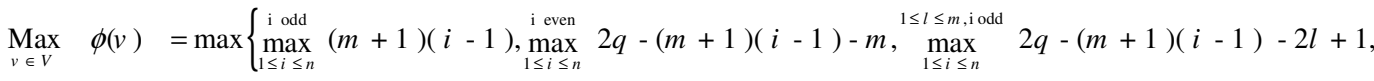

$$
\begin{aligned}
& \left.\max _{1 \leq i \leq n}^{1 \leq l \leq m, \text { ieven }} 2 q-(m+1)(i-2)+2 l\right\} \\
& =2 q-1 \text {, the maximum value of all odds. }
\end{aligned}
$$

Thus $\phi(v) \in\{0,1,2, \ldots, 2 q-1\}$.

(b) Clearly $\phi$ is a one - to - one mapping from the vertex set of $G$ to $\{0,1,2, \ldots, 2 q-1\}$.

(c) It remains to show that the labels of the edges of $G$ are all the odd integers of the interval [1, $2 q-1]$.

The range of $\left|\phi\left(v_{i}\right)-\phi\left(v_{i+1}\right)\right|=\{2 q-2 i(m+1)+m: i=1,2, \ldots, n-1\}$

$$
=\{2 q-m-2,2 q-3 m-4, \ldots, 2(q-n(m+1)+1)+3 m\}
$$

The range of $\left|\phi\left(v_{i}\right)-\phi\left(v_{\mathrm{i}}^{1}\right)\right|=\{2 q-2(m+1)(i-1)-2 l+1:($ o odd $) ; 1 \leq i \leq n ; 1 \leq l \leq m\}$

$$
=\{2 q-2 l+1,2(q-(m+1)-l)+1, \ldots, 2(q-m n-n)+3\}
$$

The range of $\left|\phi\left(v_{i}\right)-\phi\left(v_{\mathrm{i}}^{1}\right)\right|=\{2 q-(m+1)(2 i-3)-m-2 l:($ i even $) ; 1 \leq i \leq n ; 1 \leq l \leq m\}$

$=\{2(q-m-l)-1,2(q-3 m-l)-5, \ldots, 2 q-(m+1)(2 n-3)-3 m\}$

Hence $\{|\phi(u)-\phi(v)|: u v \in E\}=\{1,3,5, \ldots, 2 q-1\}$ so that the graph $G=P_{n}$ e $m K_{1}$ is odd graceful.

\section{Concluding Remarks}

Graceful and odd gracefulness of a graph are two entirely different concepts. A graph may posses one or both of these or neither. In the present work we investigate four new families of odd graceful graphs. To investigate similar results for other graph families and in the context of different labeling techniques is an open area of research.

\section{Open Problems}

1-Let $G$ be the linear $k C_{8}$-snake with $m$-pendant edges . Is $G$ odd graceful ?

2-Let $G$ be the linear $k C_{n}$-snake with $m$-pendant edges ( $n$ even). Is $G$ odd graceful ? 
International journal on applications of graph theory in wireless ad hoc networks and sensor networks (GRAPH-HOC) Vol.4, No.4, December 2012

\section{References}

[1] R. B. Gnanajothi, Topics in Graph Theory, Ph.D. Thesis, Madurai Kamaraj University, (1991).

[2] E.M.Badr, M. I. Moussa and K. Kathiresan, Crown graphs and subdivision of ladders are odd graceful, International Journal of Computer Mathematics, vol.88,Issue 17, PP. 3570- 3576 (2011)

[3] G. Chartrand and L. Lesniak, Graphs and Digraphs, Chapman and hall/CRC, Boca Raton, London, New York, Washinton, D. C., 1996.

[4] C. Barrientos, Difference Vertex Labelings, Ph. D. Thesis, University Politecnica De Catalunya, Spain, 2004.

[5] A. Rosa, Cyclic Steiner Triple Systems and Labelings of Triangular Cacti, Scientia, 5, (1967), 87-95.

[6] E. M. Badr and M. I. Moussa, Odd Graceful Labelings of Syclic Snakes, 1st International Conference on Mathematics and Statistics, Sharjah, E. U. A, (2010).

\section{Author}

El-Sayed Badr received the B.Sc. (Mathematics) the M.Sc. (Pure Mathematics) degree from Benha University, Benha, Egypt in 1994, 2000 respectively. Also, he received Ph.D. ( Computer Science) degree from Department of Applied Informatics, Macedonia University, Thessaloniki, Greece, in 2006. He is a University lecturer of Computer Science with the Benha University, Faculty of Science, Department of Mathematics. His current research is Parallel Programming, linear programming and Graph Theory.

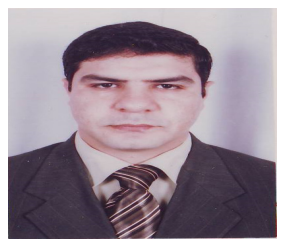

